Instituto Internacional de Investigación y Desarrollo Tecnológico Educativo INDTEC, C.A.

DOI: https://doi.org/10.29394/Scientific.issn.2542-2987.2021.6.21.11.220-235

OAl-PMH: http://www.indteca.com/ojs/index.php/Revista Scientific/oai

Artículo Original / Original Article

\title{
Resiliencia familiar frente a la conducta de riesgo de los adolescentes, Comunidad "El Blanco"
}

Autores: Sandra Auxiliadora Romero Chávez Universidad Técnica de Manabí, UTM sandra.romero@utm.edu.ec Portoviejo, Ecuador https://orcid.org/0000-0003-3512-8197

Xiomara Isabel Chinga Demera Universidad Técnica de Manabí, UTM xchinga3152@utm.edu.ec

Portoviejo, Ecuador https://orcid.org/0000-0002-7382-3046

\section{Resumen}

La adolescencia es una etapa de vida marcada por una serie de cambios físicos, psicológicos y sociales, que impulsa individuo a tomar decisiones propias sobre cómo pensar, sentir o actuar, exponiéndolos a ambientes adversos donde se despliegan diversas maneras para incurrir en conductas de riesgo como consumo de drogas y bebidas alcohólicas, embarazo precoz, delincuencia, entre otros, cuyas consecuencias podrían ser superados con el apoyo de la familia. Este estudio tiene como objetivo identificar la influencia de la resiliencia familiar frente a las conductas de riesgos de los adolescentes, Comunidad "EI Blanco". El proceso metodológico fue de tipo descriptivo-analítico apoyado en un enfoque cuantitativo, se aplicó la técnica de encuesta a una población de 100 adolescentes, al presidente del sector, 6 líderes barriales y 50 padres de familia escogidos aleatoriamente. Se comprobó que, en la comunidad existe un alto nivel de resiliencia familiar frente a las conductas de riesgo en las que se ven involucrados los adolescentes, ya que poseen capacidades para resistir y superar los problemas surgidos, además de adaptarse a las situaciones de crisis. Es necesario el fortalecimiento de las relaciones familiares debido a que el adolescente percibe poco grado de afecto y de comunicación en este contexto.

Palabras clave: adolescencia; conductas de riesgo; resiliencia familiar. Código de clasificación internacional: 6114.03 - Comportamiento colectivo.

Cómo citar este artículo:

Romero, S., \& Chinga, X. (2021). Resiliencia familiar frente a la conducta de riesgo de los adolescentes, Comunidad "EI Blanco". Revista Scientific, 6(21), 220-235, e-ISSN: 2542-2987. Recuperado de: https://doi.org/10.29394/Scientific.issn.2542-2987.2021.6.21.11.220-235

Fecha de Recepción: 23-03-2021
Fecha de Aceptación: 06-07-2021
Fecha de Publicación: 05-08-2021 
OAl-PMH: http://www.indteca.com/ojs/index.php/Revista Scientific/oai

Artículo Original / Original Article

\title{
Family resilience in the face of adolescent risk behavior, "El Blanco" Community
}

\begin{abstract}
Adolescence is a stage of life marked by a series of physical, psychological and social changes, which prompts individuals to make their own decisions about how to think, feel or act, exposing them to adverse environments where various ways are deployed to engage in risky behaviors such as consumption of drugs and alcoholic beverages, early pregnancy, delinquency, among others, the consequences of which could be overcome with the support of the family. The objective of this study is to identify the influence of family resilience in the face of risk behaviors of adolescents, "EI Blanco" Community. The methodological process was descriptive-analytical based on a quantitative approach, the survey technique was applied to a population of 100 adolescents, the president of the sector, 6 neighborhood leaders and 50 randomly chosen parents. It was found that, in the community, there is a high level of family resilience in the face of risk behaviors in which adolescents are involved, since they have capacities to resist and overcome the problems that have arisen, in addition to adapting to crisis situations. It is necessary to strengthen family relationships because the adolescent perceives little degree of affection and communication in this context.
\end{abstract}

Keywords: adolescence; risk behaviors; family resilience. International classification code: 6114.03 - Collective behavior.

\footnotetext{
How to cite this article:

Romero, S., \& Chinga, X. (2021). Family resilience in the face of adolescent risk behavior, "EI Blanco" Community. Revista Scientific, 6(21), 220-235, e-ISSN: 2542-2987. Recovered from: https://doi.org/10.29394/Scientific.issn.2542-2987.2021.6.21.11.220-235
}

Date Received: 23-03-2021
Date Acceptance: 06-07-2021
Date Publication: 05-08-2021 


\section{Introducción}

La resiliencia procede del verbo en latín, del término resilio, que significa volver a saltar, rebotar, resistir ante cualquier agresión; se comenzó a utilizar en el campo de la Física, a fin de calcular la capacidad de ciertos materiales resistentes que pueden recobrar su posición original cuando han soportado cargas o impactos. Según los autores Rutter (1993); Werner (2003), citados por Uriarte (2005), el término Resiliencia:

Fue incorporado en las ciencias sociales a partir de los años 60 y caracteriza la capacidad que tienen las personas para desarrollarse psicológicamente sanos y exitosos a pesa de vivir en contextos de alto riesgo, como entornos de pobreza y familias multiproblemáticas, situaciones de estrés prolongado, centros de internamiento, etc. (pág. 66).

A partir de esta premisa, se menciona que en el contexto mundial la resiliencia ha tomado importante relevancia científica, pues se ha convertido en un tema de gran impacto, mismo que ha llevado a diversos investigadores a estudiar este término desde diversas perspectivas, teorías, paradigmas, epistemologías, entre otros; todo esto, a fin de descubrir especialmente cuál o cómo es la reacción de una persona para enfrentar una determinada situación. Es importante mencionar, que la resiliencia no solo se basa en superar un problema, sino también en la fortaleza que tiene el individuo para transformar determinadas situaciones conflictivas en oportunidades, siendo entes activos de su propio bienestar.

Principalmente, el término de resiliencia se refiere al proceso de superación que permite a los individuos sobreponerse ante las adversidades y los contextos desfavorecidos en el que se encuentra inmerso. Bajo esta perspectiva, es entendida como la capacidad que poseen los individuos para contrarrestar y superar adversidades, a pesar de vivir en un contexto desfavorecido o haber experimentado situaciones conflictivas, desarrollando paulatinamente respuestas orientadas a la consecución de su bienestar y 
desarrollo humano.

Como menciona Herrera (2017a): "la vida está sujeta a constantes cambios, frente a los cuales es necesario contar con capacidades como la resiliencia [...]" (pág. 4); que describe Pasqualini (2010), cita por Herrera (2017b): como la "capacidad universal de todo ser humano de resistir ante condiciones adversas y recuperarse, desarrollando, paulatinamente, respuestas orientadas hacia la construcción de un ajuste psicosocial positivo al entorno, a pesar de la existencia simultánea de dolor y conflicto intrapsíquico" (pág. 4).

Tal es el caso, que los momentos difíciles o las situaciones traumáticas obligan al individuo hacerle frente a la realidad para no tener consecuencias devastadoras en sus vidas; por lo que ser resilientes implica a las personas, demostrar habilidades para sobreponerse a las distintas adversidades que se le presentan; dicho en otras palabras, debe ser capaz de soportar el estrés, superar traumas, resolver conflictos y sobrellevar una mala etapa en su vida.

Ahora bien, para recorrer el camino hacia la resiliencia familiar es indispensable entender todos los aspectos que caracterizan a una familia, desde sus fortalezas hasta sus debilidades, que los podrían convertir un grupo familiar capaz de identificar sus cualidades relacionales hacia la superación de sus problemas. En este sentido, García, García y Rivera (2015), manifiestan que:

La importancia del estudio sobre la capacidad que tienen los padres para hacer frente a la adversidad en alguno de sus hijos radica en la necesidad de identificar los factores involucrados en los ámbitos personal y familiar que pueden ser utilizados como herramientas en la prevención y tratamiento de la dependencia al alcohol (pág. 165).

Es preciso indicar, que la adolescencia al ser un periodo de vida de la persona, comprende la aparición de la pubertad y marca el final de la infancia, dando inicio de la edad adulta, su rango de duración varía según las diferentes 
fuentes y opiniones médicas, científicas y psicológicas. Como se estipula en el Código de la Niñez y Adolescencia (2003): "niño o niña es la persona que no ha cumplido doce años de edad. Adolescente es la persona de ambos sexos entre doce y dieciocho años de edad" (art. 4).

Para una definición más clara, Seoane (2015): manifiesta que "las conductas de riesgo son acciones voluntarias o involuntarias que ponen en peligro la integridad física y psíquica del adolescente y su entorno" (pág. 2). Se destaca, que muchas de las conductas de riesgos tienen su origen en un determinado estilo de vida de la persona, mismo que dentro de una familia está compuesto por una serie de valores, costumbres y dinámica en su funcionamiento.

Y es así que, la atención puesta en la dinámica familiar ayuda a comprender la situación que se quiere investigar por medio de este estudio. A partir de esta aportación, Gallego, Pino, Álvarez, Vargas y Correa (2019), señalan que:

En la familia se construyen las primeras experiencias vinculares del niño. Estas experiencias le proporcionan, o por lo menos se espera que le brinde, el bienestar para su desarrollo armónico e integral. Sin embargo, las dinámicas sociales, culturales, económicas y familiares han llevado a que el concepto de familia y las relaciones que se tejen en esta se transforme de generación en generación [...] (pág. 7).

Se destaca, que los adolescentes están en constante evolución, que los expone a ser vulnerables diversos factores de riesgo que podrían ser superados con el apoyo de la familia. Es así, que independiente de la época en que esta transcurre, la familia y será el lugar donde se brinda la identidad, los valores, los principios y las tradiciones a los individuos. En correspondencia a lo anteriormente manifestado, Santana (2019), dice que:

Cada familia es única, por lo que es posible que activen la resiliencia al ser receptivos y apoyar el desarrollo de estrategias de afrontamiento, por ejemplo, su capacidad de 
distinguir los cambios y problemas prácticos a los que se enfrentan; [...] En la familia, las relaciones de confianza, amor y esperanza son la base que sustenta el andamiaje de la resiliencia familiar y que permite en su interior la democracia, los derechos humanos, la justicia social, el diálogo igualitario [...] (págs. 6-7).

En este sentido, se menciona que este artículo es de carácter investigativo, pues se pretende responder de alguna manera a la siguiente interrogante: ¿Cómo influye la resiliencia familiar en las conductas de riesgos de los adolescentes?, pues con ello se persigue como objetivo describir la influencia de la resiliencia familiar frente a las conductas de riesgos a las que están expuesto los adolescentes; es así, que la importancia de esta investigación radica en la contribución de conocimientos para la sociedad en general y a su vez sirva de referencia para investigaciones futuras.

\section{Metodología}

Para la formación de este estudio se realizó una investigación de tipo descriptiva-analítica; misma que permitió realizar un estudio exhaustivo sobre la resiliencia familiar frente a las conductas de riesgos de los adolescentes en la comunidad rural "El Blanco", perteneciente a la parroquia de Charapotó del Cantón Sucre, Ecuador, durante el periodo febrero-junio del año 2021.

A nivel empírico, se hizo uso de la técnica de la encuesta, cuyo instrumento fue un cuestionario de preguntas cerradas, que fue aplicado a una población total de 100 adolescentes, escogidos aleatoriamente; además a ello se tomaron en consideración al presidente del sector, a los 6 líderes barriales y 50 padres de familia escogidos al azar, por lo que el tipo de muestreo fue probabilístico; la recopilación de los datos se hizo mediante el uso de herramientas virtuales tales como WhatsApp y correo electrónico.

Por la naturaleza de los datos, esta investigación estuvo apoyada en un enfoque cuantitativo, ya que se procesó estadísticamente la información 
obtenida a través de los informantes claves, quienes fueron la principal fuente de información y detallaron aspectos relevantes para la investigación, con relación a las variables estudiadas, entre ellas resiliencia familiar y conductas de riesgos.

\section{Resultados}

Como se ha dicho anteriormente la evolución y crecimiento constate de un individuo en su etapa de adolescencia, hace que se vuelvan más vulnerables a la exposición, involucramiento y/o práctica voluntaria e involuntaria de conductas de riesgo que ponen en peligro su integridad e incluso llegan a experimentar diversas consecuencias en sus vidas; dicho esto, la importancia de la familia tiene un gran peso en la superación de las adversidades presentadas.

Es así que, cuando se habla de resiliencia familiar, se está haciendo referencia a los procesos de resistencia y superación de las adversidades mediante el apoyo del núcleo familiar, pues es aquí donde se trata de contrarrestar los efectos negativos que han traído consigo el involucramiento en conductas de riesgo, para la recuperación, mejoramiento y consecución del bienestar físico, emocional y social del individuo.

En concordancia a lo anteriormente dicho, estudiar a las familias resilientes engloba la identificación de características propias de la persona como miembro de la unidad familiar, y como ellos se relacionan entre sí para desarrollar diversas estrategias de afrontamiento de las adversidades a las que estuvieran expuestos.

En este sentido, al hacer referencia a la comunidad investigada, los que se encuentran más vulnerables a vivenciar experiencias negativas son los adolescentes, debido a que están en constante interacción con otros individuos, tienen muchos deseos de autonomía, curiosidad e incluso quieren a como dé lugar formar parte de un determinado grupo de amigos, sin medir 
las consecuencias que esto podría desencadenar.

Es por ello, que a fin de identificar la influencia de la resiliencia familiar frente a las conductas de riesgos de los adolescentes en la Comunidad "El Blanco", es necesario en primera instancia determinar las conductas de riesgos a las que están expuestos; para ello, se aplicaron técnicas e instrumentos acorde al tipo de investigación; que mediante las encuestas aplicadas y una vez realizado el procesamiento de la información recopilada, se obtuvieron los siguientes resultados que dan cumplimiento al objetivo planteado.

A continuación, en la tabla 1 se muestran la consideración que tienen la población encuestada de la comunidad "El Blanco", con relación a las posibles conductas de riesgo en las llegan a incurrir los adolescentes en mayor o menor frecuencia.

Tabla 1. Conductas de riesgo en las que se pueden involucrar los adolescentes.

\begin{tabular}{|c|c|c|}
\hline INDICADOR & CANTIDAD & PORCENTAJE \\
\hline $\begin{array}{c}\text { Consumo de drogas y de } \\
\text { bebidas alcohólicas }\end{array}$ & 61 & $39 \%$ \\
\hline Problemas de autoestima & 6 & $4 \%$ \\
\hline Delincuencia & 44 & $28 \%$ \\
\hline Embarazo precoz & 17 & $11 \%$ \\
\hline $\begin{array}{c}\text { Adquisición de } \\
\text { enfermedades de } \\
\text { transmisión sexual }\end{array}$ & 3 & $2 \%$ \\
\hline Participación en riñas & 8 & $5 \%$ \\
\hline Prostitución & 3 & $2 \%$ \\
\hline Deserción escolar & 14 & $9 \%$ \\
\hline Total & 156 & $100 \%$ \\
\hline
\end{tabular}

Fuente: Las Autoras (2021).

Del total de las personas encuestadas, un 39\% consideran que la mayor conducta de riesgo en las que se pueden involucrar los adolescentes es en el consumo de drogas y de bebidas alcohólicas por su fácil acceso; el 28\% 
considera que es la delincuencia, en un $11 \%$ el embarazo precoz, seguido de un $9 \%$ que señalaron deserción escolar; $5 \%$ participación en riñas, $4 \%$ problemas de autoestima, mientras que de forma igualitaria al $2 \%$ adquisición de enfermedades de transmisión sexual y prostitución.

Estos hallazgos guardan relación con lo manifestado por Amar y Hernández (2005); Hernández y Lucio (2006), citados por Aguiar y AcleTomasini (2012), cuando mencionan que:

El ser adolescente en ocasiones implica estar inmerso en factores de riesgo que en cierto modo pueden afectar el buen desempeño escolar, familiar y social. Estos factores pueden darse en todos los contextos en que se desenvuelven y que involucran afectaciones, tales como trastornos de conducta, agresividad física, verbal, irresponsabilidad, riesgos suicidas y relaciones interpersonales pobres (pág. 58).

Desde esta perspectiva, se añade que, la adolescencia sería la etapa de la vida caracterizada por un sinnúmero de cambios que experimenta el individuo, llevándolo a ser vulnerable a enormes presiones que lo exponen a diversas situaciones de riesgo, mismas que son catalogadas como retos 0 desafíos que deben ser superados para determinar su propia de identidad, y no perjudicar su integridad física, psicológica y social.

Como exponen Tamayo, Miraval, Mansilla, Miraval y Tamayo (2020): "con respecto a las conductas de riesgo ocurridas en el seno familiar de los adolescentes, se encontró factores predisponentes como: riñas o pelas alguna vez, abuso sexual, intento de suicidio, consumo de mariguana, depresión [...]" (pág. 42). Es preciso indicar que, la mayoría de los adolescentes adoptan comportamientos inadecuados, que ponen en peligro su integridad e incluso hasta su vida, debido a que no siempre perciben el riesgo como tal, llevándolos a actuar como si esto no pudiera ocasionarles a ellos o a las personas que los rodean ningún daño o desencadenar un sinnúmero de consecuencias.

Todo ello confirma, que el desarrollo de nuevas ideologías, maneras de 
pensar, así como la incorporación de nuevos valores han influenciado en el comportamiento de las generaciones actuales dentro de la sociedad, generando con ello nuevos patrones de conductas en los adolescentes y una nueva organización social, en donde la familia se ve seriamente afectada.

Tabla 2. Causa principal para que los adolescentes desarrollen conductas de riesgo. Adolescentes, líderes barriales y padres de familia de la Comunidad "El Blanco".

\begin{tabular}{|c|c|c|}
\hline INDICADOR & CANTIDAD & PORCENTAJE \\
\hline Estilo de crianza & 21 & $13 \%$ \\
\hline Curiosidad & 29 & $19 \%$ \\
\hline $\begin{array}{c}\text { Quebrantamiento en la } \\
\text { homeostasis familiar }\end{array}$ & 44 & $28 \%$ \\
\hline $\begin{array}{c}\text { Padres con comportamiento } \\
\text { social inadecuado }\end{array}$ & 7 & $4 \%$ \\
\hline $\begin{array}{c}\text { Permisividad y tolerancia de } \\
\text { la conducta del adolescente }\end{array}$ & 12 & $8 \%$ \\
\hline $\begin{array}{c}\text { Pertenecer a un grupo de } \\
\text { amigos }\end{array}$ & 18 & $12 \%$ \\
\hline Rebeldía & 25 & $16 \%$ \\
\hline Total & 156 & $100 \%$ \\
\hline
\end{tabular}

Fuente: Las Autoras (2021).

La tabla 2, describe que la causa principal para que los adolescentes desarrollen conductas de riesgo son: quebrantamiento en la homeostasis familiar $28 \%$, curiosidad $19 \%$, seguido de la rebeldía con un $16 \%$, estilo de crianza $13 \%$, pertenecer a un grupo de amigos $12 \%$, permisividad y tolerancia de la conducta del adolescente $8 \%$, mientras que en un $4 \%$ es causado por los padres con comportamiento social inadecuado. Interpretando a Salas (2018):

Los comportamientos de riesgo que llevan a cabo los adolescentes entre los que se puede mencionar principalmente el alcoholismo, la drogadicción, el tabaquismo y las conductas sexuales inseguras, la mala alimentación, la depresión y el suicidio, el sedentarismo y la producción de lesiones no 
intencionales, presentan distintos procesos involucrados (pág. 11).

Por lo mencionado anteriormente, se destaca que existen diversos factores que llevan a los adolescentes a incurrir en conductas de riesgos, mismas que están relacionadas principalmente a los cambios propios de la etapa; a los valores familiares, culturales y sociales que los conducen a asumir riesgos en muchas de las decisiones que toman, y el tipo de influencia que percibe el adolescente en su contexto social sea esto dentro de la familia, las relaciones interpersonales que establecen en el lugar de estudio y el barrio donde viven. Desde esta perspectiva, Guamán (2018), añade que:

La adolescencia es una etapa en donde se encontrarán cambios y transformaciones en donde se presentarán reajustes en este periodo siendo la fase en donde se manifiestan y se revelan como seres vulnerables ante comportamiento riesgoso siendo así se debe conocer cuales o a que se denomina factores de riesgos y protección para estos púberes (pág. 9).

En este sentido, se menciona que la población juvenil se encuentra más propensa a la aparición de conductas de riesgos, debidos a los cambios surgidos en esta etapa, mismos que están acompañados por el desequilibrio emocional propio de su edad, el deseo de independencia y de formar parte de un grupo de amigos, la formación de su personalidad, entre otros aspectos. Es así, que las conductas de riesgo que desarrollan los jóvenes, se presentan en un proceso progresivo que se relaciona poco a poco y que se va agravando, cuando el adolescente está más preparado para llevar a cabo acciones por cuenta propia.

Pero es indudable mencionar, el rol que cumple la familia en este tipo de situaciones pues es y ha sido siempre fuente de bienestar, satisfacción y aprendizaje para todos sus integrantes; por lo que la población encuestada considera que la familia sí es un pilar fundamental para afrontar los desafíos 
que se le presenta, pues mencionan que si un miembro del hogar desarrolla algún tipo de conductas de riesgo lo resolverían en familia, lo que llega a permitir a los adolescentes reconocer la fortaleza más allá de la vulnerabilidad y tener conciencia sobre todo en la posibilidad de transcender.

\section{Conclusiones}

Los resultados de la investigación evidenciaron que la adolescencia al ser una etapa de desarrollo, donde el individuo experimenta un sinnúmero de cambios en su apariencia física y en sus emociones, hace una demanda creciente de autonomía que en momentos ocasionan desacuerdos en relación sobre las que los padres quieren seguir ejerciendo control sobre ello.

Por lo que, el adolescente que se vea involucrado en alguna situación que amenace su integridad debe poseer capacidades para enfrentar, sobreponerse y vencer las adversidades; pero sin duda el afrontamiento dependerá del equilibrio que consiga cada persona en su situación y circunstancia. Se hace relevante destacar la importancia que tienen los dominios de la familia en la vida de las personas, pues al ser la primera institución sociabilizadora del individuo, debe fortalecer la resiliencia en los adolescentes, estimulando la parte afectiva, la reciprocidad, el amor, para que el individuo desde cada etapa de su vida vaya superando sus propias adversidades y pueda reconocer en quienes puede apoyarse o a quienes acudir.

Es necesario mencionar, que en la comunidad rural investigada a pesar de que el adolescente percibe poco grado de afecto y de comunicación dentro del hogar, existe un alto nivel de resiliencia familiar frente a las conductas de riesgo en las que se ven involucrados los adolescentes, pues los padres ofrecen apoyo incondicional a sus hijos y tratan de solucionar los problemas surgidos, a pesar de que incurran en actos delictivos o situaciones que ponen en peligro su integridad. 
Por todo lo dicho, la familia como unidad también necesita desarrollar estas capacidades de resiliencia, porque a lo largo de su proceso cambiante, llegarán ocasiones en las que la frustración amenace con ocasionar un quebrantamiento en la homeostasis familiar; todo ello demuestra, que es muy significativo continuar con investigaciones sobre este tema, pues es necesario sensibilizar a la ciudadanía sobre la influencia familiar en el desarrollo integral de los adolescentes y la definición de sus patrones de conductas.

\section{Referencias}

Aguiar, E., \& Acle-Tomasini, G. (2012). Resiliencia, factores de riesgo y protección en adolescentes mayas de Yucatán: Elementos para favorecer la adaptación escolar. Acta Colombiana de Psicología, 15(2), 53-64, e-ISSN: 0123-9155. Recuperado de:

https://www.redalyc.org/articulo.oa?id=79825836002

Código de la Niñez y Adolescencia (2003). Los Niños, Niñas y Adolescentes como sujetos de Derechos. Ley No. 2002-100. Ecuador: Congreso Nacional.

Gallego, A., Pino, J., Álvarez, M., Vargas, E., \& Correa, L. (2019). La dinámica familiar y estilos de crianza: pilares fundamentales en la dimensión socioafectiva. Hallazgos, 16(32), 1-20, e-ISSN: 1794-3841. Recuperado de:

https://www.redalyc.org/articulo.oa?id=413859265008

García, J., García, M., \& Rivera, S. (2015). Potencial resiliente en familias con adolescentes que consumen y no consumen alcohol. Acta Colombiana de Psicología, 18(2), 163-172, e-ISSN: 1909-9711. Recuperado de: https://doi.org/10.14718/ACP.2015.18.2.14

Guamán, L. (2018). La familia como factor de riesgo para los adolescentes que consumen sustancias psicoactivas de 14 a 17 años del CDID. Tesis. Guayaquil, Ecuador: Universidad de Guayaquil. Recuperado de: 
http://repositorio.ug.edu.ec/handle/redug/26768

Herrera, J. (2017a,b). Resiliencia y su relación con el entorno familiar en los estudiantes de bachillerato del ISTDAB de la ciudad de Loja en el periodo septiembre 2015-julio 2016. Tesis. Loja, Ecuador: Universidad Nacional de Loja. Recuperado de: https://dspace.unl.edu.ec/ispui/handle/123456789/19520

Salas, F. (2018). Caracterización de factores implicados en las conductas de riesgo en adolescentes. Abra, 38(56), 1-16, e-ISSN: 2215-2997. Recuperado de: https://doi.org/10.15359/abra.38-56.3

Santana, E. (2019). La construcción de la resiliencia familiar en la experiencia de la discapacidad: una posibilidad para generar procesos inclusivos. Sinéctica, (53), 1-23, e-ISSN: 2007-7033. Recuperado de:

https://sinectica.iteso.mx/index.php/SINECTICA/article/view/965

Seoane, A. (2015). Adolescencia y Conductas de Riesgo. Trabajo final de grado. Montevideo, Uruguay: Universidad de la República. Recuperado de: https://hdl.handle.net/20.500.12008/7524

Tamayo, M., Miraval, Z., Mansilla, P., Miraval, L., \& Tamayo, M. (2020). La dinámica familiar asociada a conductas de riesgo en adolescentes de las familias de la jurisdicción de Aparicio Pomares. Huánuco, Perú. ReHuSo: Revista de Ciencias Humanísticas y Sociales, 5(3), 3743, e-ISSN: 2550-6587. Recuperado de:

https://revistas.utm.edu.ec/index.php/Rehuso/article/view/2599

Uriarte, J. (2005). La resiliencia. Una nueva perspectiva en psicopatología del desarrollo. Revista de Psicodidáctica, 10(2), 61-79, e-ISSN: 11361034. Recuperado de:

https://www.redalyc.org/articulo.oa?id=17510206 


\section{Sandra Auxiliadora Romero Chávez}

e-mail: sandra.romero@utm.edu.ec

Nacida en Manabí, Ecuador, el 14 de mayo del año 1969.

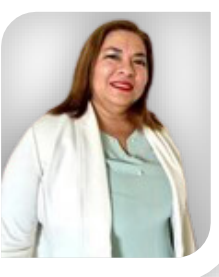

Magister en Educación y Desarrollo Social por la Universidad Tecnológica Equinoccial (UTE); Licenciada en Trabajo Social por la Universidad Técnica de Manabí (UTM); Docente Auxiliar 2 a tiempo completo del departamento de Ciencias Sociales y del Comportamiento; Carrera de Trabajo Social y responsable del departamento de Práctica Preprofesional de la Facultad de Ciencias Humanística y Sociales de la Universidad Técnica de Manabí (UTM). 


\section{Xiomara Isabel Chinga Demera}

e-mail: xchinga3152@utm.edu.ec

Nacida en Manabí, Ecuador, el 23 de agosto del año

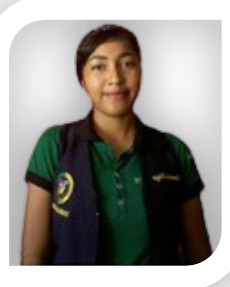
1998. Egresada en la carrera de Trabajo Social en modalidad presencial de la Universidad Técnica de Manabí (UTM); me desempeñé como asistente de los Técnicos responsables de la Misión "Mis Mejores Años" (GAD Charapotó); he participado en los Encuentros Internacionales de Resiliencia organizados por la Red de Resiliencia del Ecuador; realicé cursos sobre técnicas para la organización de fiestas y eventos. 